\title{
Análisis de las consecuencias de la desfinanciación de medicamentos del 1 de septiembre de 2012
}

\author{
Ignacio Compairé Berguaa ${ }^{1}$, Alberto Compairé Berguaa ${ }^{1}$, José Antonio Arner Navarro², \\ Daniel García Lerma ${ }^{3}$, Agustín Ramón Gazo ${ }^{4}$ \\ 1. Farmacéutico comunitario en Huesca. 2. Farmacéutico comunitario en Ejea de los Caballeros (Zaragoza). 3. Farmacéutico comunitario en Zaragoza. \\ 4. Farmacéutico comunitario en Biescas (Huesca).
}

\section{PALABRAS CLAVE}

dispensación, desfinanciación de medicamentos, adherencia, accesibilidad, farmacia comunitaria

\section{ABREVIATURAS}

FADSP: Federación de Asociaciones para la Defensa de la Sanidad Pública.

ISFAS: Instituto Social de las Fuerzas Armadas. MUFACE: Mutualidad de Funcionarios de la Administración Civil del Estado.

OTC: over the counter. RD: Real Decreto. SNS: Sistema Nacional de Salud.

TSI: tarjeta sanitaria individual.

\section{KEYWORDS}

dispensation, defunding of medicines, adherence, accessibility, community pharmacy

\section{RESUMEN}

Objetivos: Conocer si la financiación de algunos medicamentos limita su acceso, en especial a las personas con rentas más bajas, valorar la adherencia de los pacientes a dichos medicamentos y analizar el conocimiento de la población sobre dicha medida.

Métodos: Se realizó un estudio multicéntrico en cuatro farmacias comunitarias aragonesas, considerándose la dispensación o no del medicamento como variable dependiente, y se recogieron una serie de variables generales (edad, sexo, especialidad, precio, grupo terapéutico, código de aportación, información y tratamiento crónico o de inicio). Se utilizó una tabla de contingencia entre la dispensación o no de los medicamentos y el TSI de cada paciente y se le aplicó el test de chi-cuadrado $\left(\chi^{2}\right)$.

Resultados: Fueron dispensados 279 de 316 medicamentos, con una participación activa del farmacéutico. El 59,8\% de los pacientes había sido informado de la medida previamente. Se encontró asociación estadística $(p=0,022)$ entre la dispensación o no del medicamento y los porcentajes de aportación TSI002 y TSI003. Un 86\% de los pacientes con tratamiento crónico continuaron con el mismo.

Discusión: Se ha demostrado que los pacientes pensionistas, que presumiblemente disponen de rentas más bajas, están más limitados a la hora de retirar estos medicamentos ya que rechazan en mayor proporción pagar su importe íntegro. El número de pacientes, sobre todo activos, dispuestos a asumir el pago de los medicamentos fue mayor que los que no aceptaron la dispensación. El papel del farmacéutico se ha revelado fundamental para conseguir el cumplimiento de la adherencia a los medicamentos.

Analysis of the consequences of the defunding of medicines of 1 September 2012

\section{ABSTRACT}

Objectives: To determine whether patient contribution to funding limits access to certain medicines, particularly among people with lower income levels; assess patient adherence to such medicines; and analyze population knowledge of the contribution to funding measures.

Methods: A multicenter study was made in four community pharmacies in Aragón (Spain), considering drug dispensation or non-dispensation as the dependent variable, with the collection of a series of general data (age, gender, drug specialty, price, therapeutic group, funding contribution code (TSI), information and chronic or starting treatment). Contingency tables contrasting dispensation or non-dispensation and the TSI of each patient were used, with application of the chi-squared test $\left(\chi^{2}\right)$.

Results: A total of 279 of 316 medicines were dispensed, with active participation of the pharmacist. A little over one-half of the patients (59.8\%) had been previously informed of the measure. A statistically significant association $(p=0.022)$ was found between dispensation or non-dispensation and the contribution codes (percentages) TSI002 and TSI003. Eighty-six percent of the patients receiving chronic treatment continued such treatment.

Discussion: It has been shown that pensioners, with presumably lower income levels, have more limited access to these medicines, since a larger proportion of these people reject having to pay the full cost of the medication. The number of patients (particularly those still active) who are willing to pay for the medicines is greater than the number who do not accept dispensation. The role of the pharmacists has been shown to be crucial in securing adherence to the medications.

El estudio fue presentado en formato póster en el V Congreso Nacional de Farmacéuticos Comunitarios celebrado en Barcelona del 15 al 17 de noviembre de 2012. Algunos de sus resultados se publicaron en un artículo redactado por Correo Farmacéutico para su número del $10 \mathrm{al}$ 16 de diciembre de 2012.

Recibido: 2-8-2013

Aceptado: 5-1-2014

Disponible online: 1-6-2014
Financiación: Ninguna ajena.

Conflicto de intereses: Los autores declaran la no existencia de conflictos de intereses en relación con el contenido del presente articulo. Cite este artículo como: Compairé I, Compairé A, Arner JA, Garcia D, Ramón A. Análisis de las consecuencias de la desfinanciación de medicamentos del 1 de septiembre de 2012. Farmacéuticos Comunitarios. 2014 Jun 01;6(2):5-10. doi:10.5672/FC.2173-9218.(2014/Nol6).002.02 Autor para correspondencia: Ignacio Compairé Bergua (icompaire@alumni.unav.es).

ISSN 1885-8619 OSEFAC (Sociedad Española de Farmacia Comunitaria). Todos los derechos reservados. 


\section{Introducción}

El 1 de septiembre de 2012 entró en vigor del Real Decreto-ley 16/2012 (1) por el cual 417 medicamentos fueron excluidos de la prestación farmacéutica en el Sistema Nacional de Salud con el objetivo de ahorrar dinero y poder garantizar la sostenibilidad de dicho Sistema. Los motivos por los que dichos medicamentos quedaron fuera de la financiación fueron varios: convivencia con un medicamento sin receta con la que comparte principio activo y dosis, establecimiento de precios seleccionados, consideración del medicamento como publicitario en nuestro entorno europeo, principio activo con un perfil de seguridad y eficacia favorable y suficientemente documentado a través de años de experiencia y uso extenso, y como principal causa estar indicados en el tratamiento de sintomas menores.

La desfinanciación de medicamentos no es una situación nueva en España ni en Europa. En 1993, en nuestro país, se reguló la selección de medicamentos reembolsados por el Sistema Nacional de Salud, desfinanciando 740 productos. Más tarde, en 1998 con el RD 1663/1998 se amplió la relación de medicamentos a otros 834 artículos más. A nivel europeo, el caso más reciente de desfinanciación extensa de medicinas lo podemos encontrar en Francia en 2006. El sistema sanitario francés establece una revisión del precio y del nivel de reembolso cada 5 años en función de una evaluación de los productos (Service Médical Rendu). A raíz de la creación de este sistema de evaluaciones de fármacos, se realizó un proceso de desfinanciación en varias fases: una en septiembre 2003 afectando a 60 productos y otra en marzo de 2006 incluyendo 156 más. Además, en diciembre de 2011 y marzo de 2012, también se vieron afectadas 26 y 20 moléculas respectivamente. En el caso francés, los fármacos afectados pasaron a no requerir prescripción médica o fármacos OTC, cosa que no ha ocurrido en nuestro país (2).

En España todavía solo se ha realizado un estudio (3), posterior al nuestro, para analizar las consecuencias de esta última medida, por lo que consideramos que los resultados mostrados en el presente artículo pueden resultar de interés.

El objetivo principal del estudio es conocer si la desfinanciación de algunos medicamentos limita su acceso, en especial a las personas con rentas más bajas. Además, se pretende valorar la adherencia de los pacientes a dichos medicamentos y analizar el conocimiento de la población sobre dicha medida, previo a su visita a la farmacia comunitaria.

\section{Métodos}

Se realizó un estudio multicéntrico observacional entre el 3 de septiembre y el 26 de octubre de 2012 en cuatro farmacias comunitarias de Aragón, dos de ellas de ámbito rural y dos urbanas.

Los datos se recogieron en una tabla diseñada ad hoc (tabla 1), previa formación del personal participante en los diferentes centros.

En el caso de que el paciente rechazase pagar la totalidad del medicamento se le argumentaba la importancia del cumplimiento para la efectividad del tratamiento.

Como límites al estudio, se tuvo en cuenta que en los primeros meses de la puesta en marcha del Real Decreto-ley no estaba totalmente regulado qué medicamentos podían seguir siendo dispensados con cargo al Sistema Nacional de Salud, por lo que las excepciones o prescripciones para determinadas indicaciones, así como los medicamentos que todavía por ser prescritos en una receta del Sistema

Tabla 1 Hoja de registro de datos

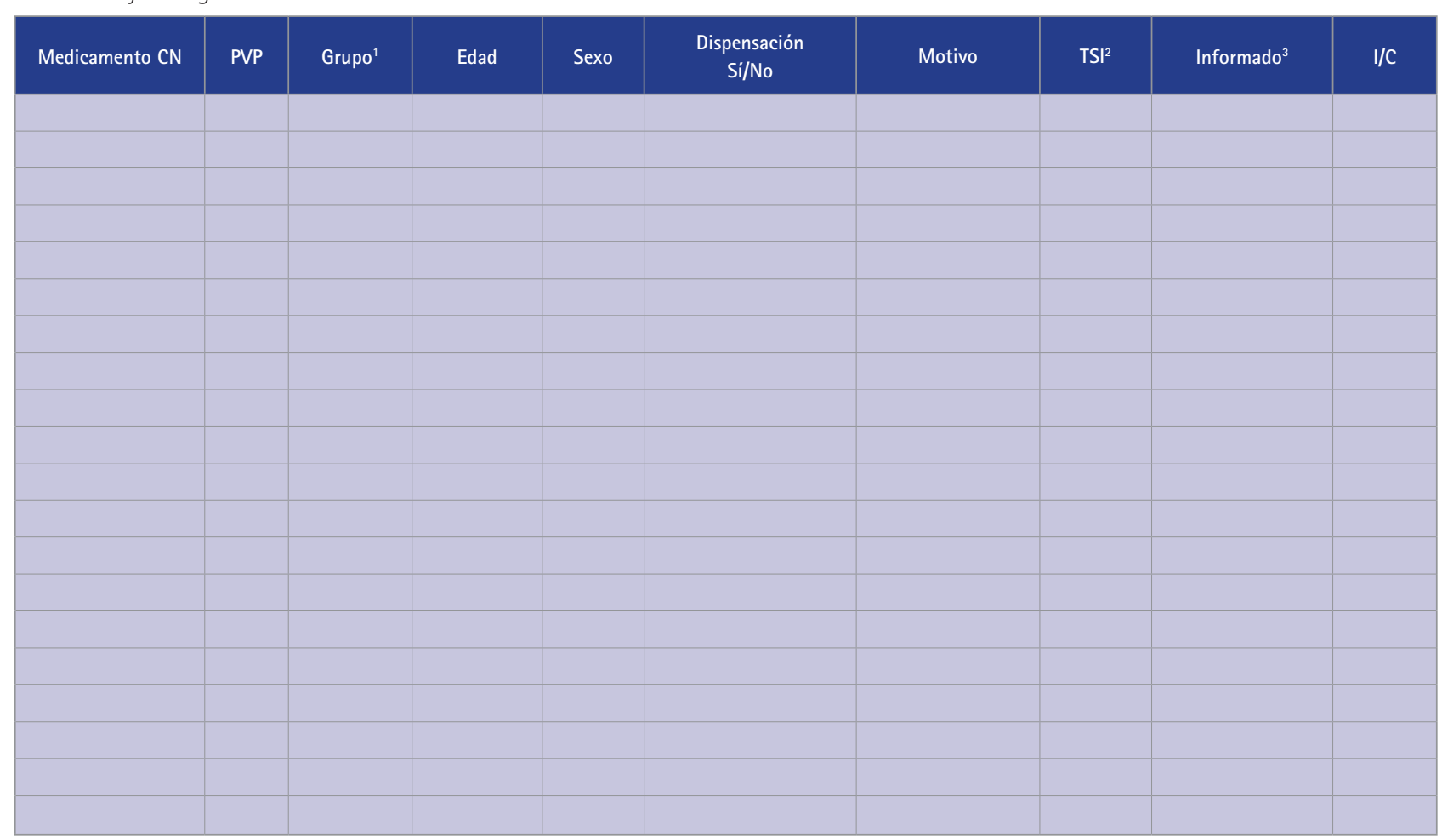

1. Grupo terapéutico al que pertenece el medicamento.

2. Porcentaje de aportación del paciente en otros medicamentos financiados.

3. El paciente conoce o no que el medicamento no está financiado previamente a la visita a la farmacia. 
Nacional de Salud, ISFAS o MUFACE no eran pagados íntegramente por el paciente, fueron excluidas del estudio. Por otro lado, algunos pacientes acudían a las farmacias con recetas blancas privadas, dificultando en algunos casos, la tarea de registrar el número de TSI, por lo que no se incluyeron. El TSI de aportación está relacionado con el nivel de renta, según datos del Servicio Aragonés de Salud, la Seguridad Social y Hacienda.

Se consideró la adherencia (dispensación o no del medicamento) como variable dependiente y el resto de variables se utilizaron para su posterior análisis mediante el paquete estadístico SPSS ${ }^{\circledR}$ versión 15.0.

Para aceptar o rechazar la hipótesis principal se utilizó una tabla de contingencia entre la dispensación o no de los medicamentos no financiados y el TSI de cada paciente y se le aplicó el test de chi-cuadrado $\left(\chi^{2}\right)$. Finalmente, solo se tuvieron en cuenta los porcentajes de aportación TSI002 y TSI003 para realizar la prueba estadística, ya que el resto de códigos fueron excluidos del análisis debido a que de otro modo, la prueba estadística resultaba inválida de acuerdo con la regla de Cochrane. También se buscó asociación estadística entre la aportación y la adherencia al tratamiento.

Se separaron los medicamentos en tres grupos: precio bajo, medio y alto, usando como puntos de corte los percentiles $33(3,12 €)$ y $67(5,73 €)$, para conocer si el importe influía en la dispensación. Por último se evaluó la existencia de diferencias en la dispensación entre las farmacias rurales y urbanas.

\section{Resultados}

Durante los dos meses que transcurrió el estudio se dispensaron 279 de un total de 316 medicamentos, lo que supuso un 88,3\% de dispensaciones. La mayoría fueron solicitados por mujeres $(64,6 \%)$ con un rango de edad predominante de más de 65 años (188 personas).

La cantidad media pagada por paciente y medicamento fue de $6,25 €$. El medicamento más caro costó al paciente $68,69 €$ y el más barato $1,72 €$. Solo tres medicamentos tenían un valor mayor de $10 €$ aunque supusieron 20 dispensaciones, como se puede observar en la figura 1. Al comparar mediante el test de chi-cuadrado, el precio medio, bajo o alto con la dis-

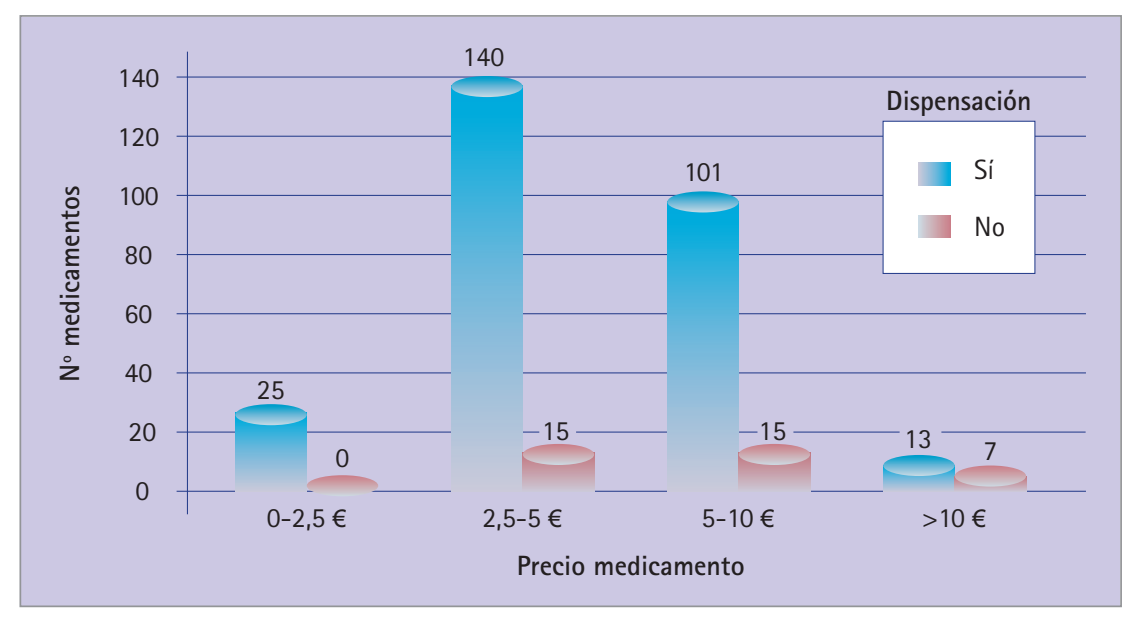

Figura 1 Distribución de medicamentos por precio y dispensación

pensación o no de los medicamentos la asociación no resultó significativa $(p=0,092)$.

El fármaco que se dispensó en más ocasiones fue Daflon ${ }^{\circledR}$ con 29 veces, seguido de Flumil Forte ${ }^{\circledR}$ (17), Artific ${ }^{\circledR}$ (16), Viscofresh ${ }^{\circledR} 0,5 \mathrm{mg}$ (15) y Acetilcisteína $200 \mathrm{mg}$, Voltaren Emulgel ${ }^{\circledR}$ y Pectox Lisina ${ }^{\circledR}$, con 14 . Unificando por grupos terapéuticos, predominaron los pertenecientes al aparato respiratorio, al cardiovascular y al tracto alimentario y metabolismo, tal como se presenta en la figura 2 .

Se encontró asociación estadística $(p=0,022)$ entre la dispensación o no del medicamento y los porcentajes de aportación TSI002 y TSI003. En la figura 3 se muestra el número de medicamentos dispensados o no, en función del código de aportación, y por lo tanto, del nivel de renta del paciente.

Por otro lado, no se encontró asociación estadística $(p=0,079)$ entre la dispensación y la continuación del tratamiento, aunque sí entre

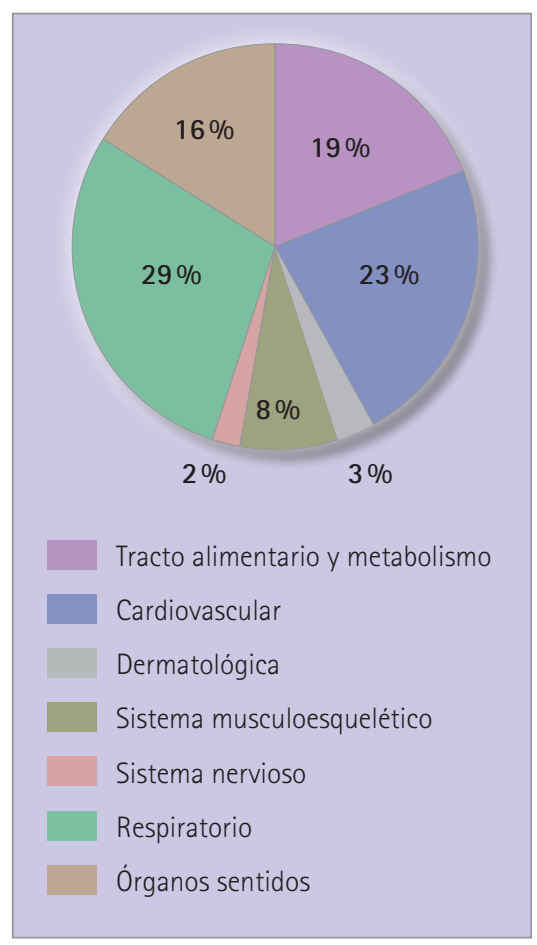

Figura 2 Porcentaje de medicamentos según el grupo terapéutico

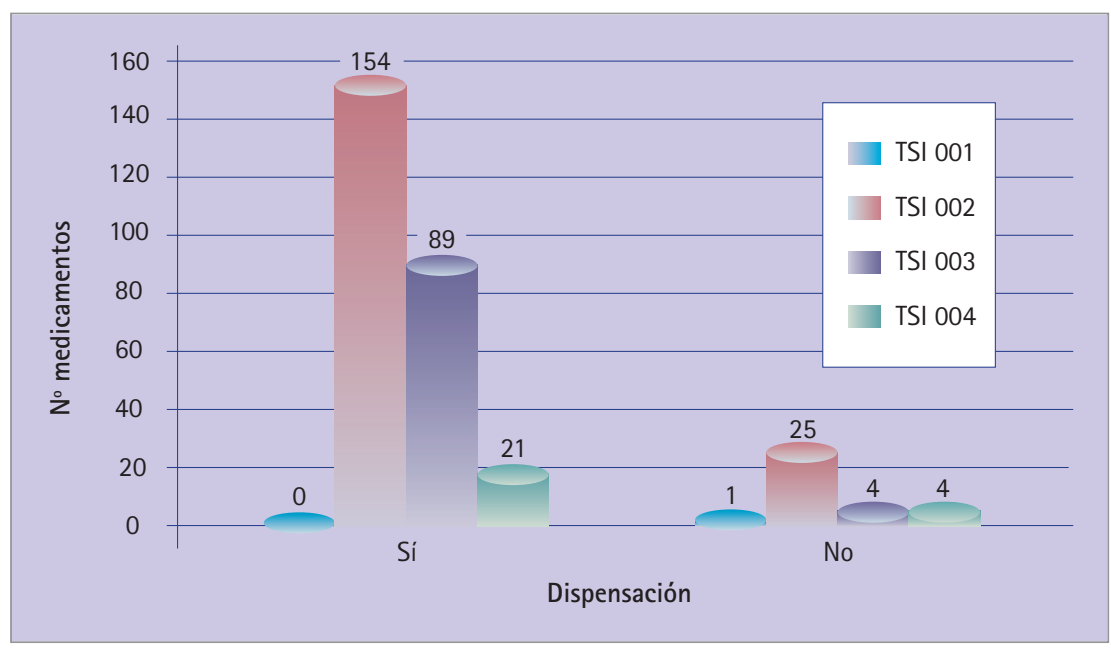

Figura 3 Dispensación de medicamentos en función del nivel de renta del paciente 


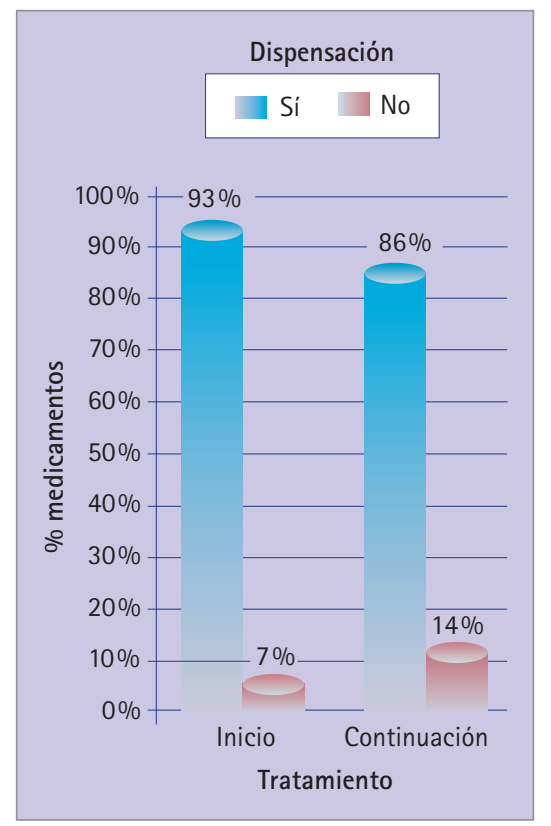

Figura 4 Distribución de los medicamentos por tipo de tratamiento y dispensación

la dispensación y la ubicación rural o urbana de la farmacia $(p<0,001)$. Sin embargo, descartando los medicamentos de precio superior, debido a que su registro estaba asociado generalmente a las farmacias urbanas, se realizó por separado un análisis de chi-cuadrado entre los datos de las farmacias urbanas y la dispensación, que resultó no significativo ( $p=0,0746)$; y una prueba exacta de Fisher entre los obtenidos en las farmacias rurales y la dispensación, con el mismo resultado $(p=0,188)$.

Solo 90, de los 316 medicamentos, eran prescripciones de inicio, y un elevado porcentaje de los pacientes con tratamiento crónico (86\%),

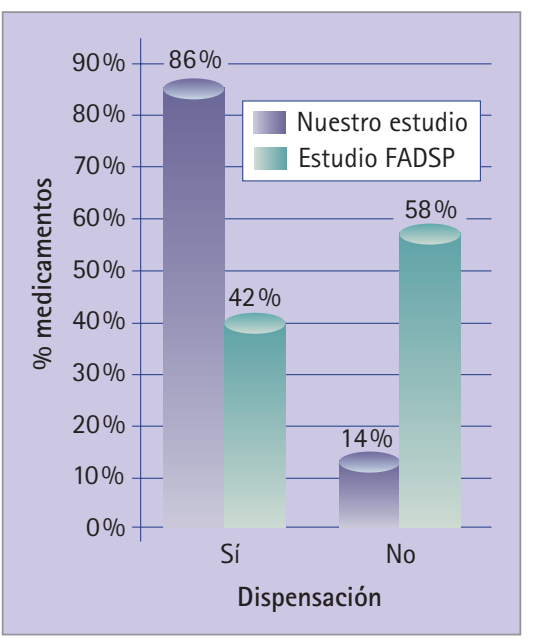

Figura 5 Grado de adherencia de los pacientes crónicos a los medicamentos desfinanciados reflejado en las figuras 4 y 5, continuaron con el mismo.

Más del 40\% de los pacientes desconocía al llegar a la farmacia que tenía que pagar íntegramente el medicamento. Los que sí lo conocían habian sido informados previamente por el médico, los medios de comunicación o por el farmacéutico en una anterior dispensación.

\section{Discusión}

Estimamos que podría considerarse una limitación del estudio el reducido margen de tiempo disponible, condicionado a la fecha del V Congreso Nacional de Farmacéuticos Comunitarios, debido a que los resultados del estudio se presentaron en formato póster entre los días 15 y 17 de noviembre de 2012 en Barcelona. Además, tuvieron que excluirse las dispensaciones en las que no se conocía el porcentaje de aportación y las consideradas como excepciones por el Sistema Nacional de Salud.

Cabe destacar que la mayoría de los pacientes a los que les fueron prescritos estos medicamentos desfinanciados, aportaban el 100\% de su valor en muchos casos, gracias a una participación activa del farmacéutico, realizando cometidos importantes de información y conciliación.

Una característica de los pacientes es que un alto porcentaje tenía más de 65 años, como era previsible, ya que se ha demostrado en numerosos estudios (4) que la mayoría de los pacientes que demandan un medicamento en las farmacias comunitarias son pensionistas. Asimismo, también coincide con otros estudios (5) en que la mayoría de las dispensaciones fueron solicitadas por mujeres.

Cuando se llevó a cabo el estudio, en la mayoría de los casos el precio de los fármacos desfinanciados todavía no se había visto aumentado. Sin embargo, con posterioridad a la realización del estudio, 65 de ellos han variado sus precios al alza. No puede pasarse por alto que el paso a fármaco desfinanciado se acompaña generalmente de un incremento considerable del precio ya que a partir de entonces el volumen de ventas del laboratorio desciende y no es el Ministerio de Sanidad el que lo determina, sino los propios laboratorios, compensando con esta medida la reducción del número de ventas. Aun así, se demostró que el precio de los medicamentos influyó en su dispensación. Los fármacos de alto importe se retiraron en menor proporción que los de bajo y medio.

La subida media de los medicamentos afectados es de más del $50 \%$, oscilando entre un 30 y un 184\%. Antes de dicha subida, el importe de muchos medicamentos se encontraba por debajo de $5 €$, pero ahora en muchos casos lo superan, lo que supone un notable aumento del gasto para el paciente y que hará imposible, en ocasiones, su dispensación y administración. Comparando estos datos con los obtenidos en Francia, dónde se mostró un aumento medio del precio del medicamento del $43 \%$ después de la desfinanciación (6), se observa un incremento algo superior en nuestro país. Por lo tanto, habrá que seguir atentos sobre todo a la evolución de los precios de los medicamentos que todavía no han sido modificados.

Además, recientemente, se ha aprobado que una serie de medicamentos, financiados con cargo al Sistema Nacional de Salud (SNS) exclusivamente para determinadas indicaciones establecidas, y conocidos como medicamentos del listado de excepciones, mantengan el precio de comercialización con cargo al SNS (precio de facturación), aunque para su comercialización fuera del SNS tengan asignados un precio distinto y más caro llamado precio notificado. Esto supone otro problema para las farmacias que cuando facturen estos medicamentos al SNS lo harán a un precio menor al que lo compraron a los almacenes de distribución mayorista con la consiguiente pérdida económica. Se está estudiando la creación de un fondo de compensación por parte de la industria farmacéutica, pero habrá que esperar hasta que se ponga en marcha, y mientras tanto, se pondrá en riesgo la supervivencia de muchas farmacias españolas.

Como se ha reflejado anteriormente, el estudio se llevó a cabo en los meses de septiembre y octubre, durante los cuales las temperaturas todavía eran algo elevadas y creemos que por ello el fármaco más dispensado resultó ser un venotónico. En muchos casos, se trataba de la última dispensación de este medicamento y de los de su grupo ya que el médico los había prescrito solo durante los meses de verano. Sin embargo, destaca 
también el número de dispensaciones de mucolíticos y expectorantes en estos meses, siendo el grupo terapéutico más demandado al asociarlo a los antitusivos. Se identificó como motivo principal de tales dispensaciones el cambio de estación, que favorece la aparición de los primeros resfriados. De forma que, si el estudio hubiese continuado más tiempo, la cantidad de mucolíticos y antitusivos se habría visto probablemente incrementada y los venotónicos habrian descendido. En cuanto a grupos como lágrimas artificiales y laxantes presuponemos un consumo constante con independencia del período estacional en el que nos encontremos. A este respecto, cabe destacar un estudio llevado a cabo en Francia en el que el número de atenciones médicas a pacientes con problemas respiratorios agudos a los que se prescribían, antes de la intervención, mucolíticos y expectorantes fue del 20-25\% y la desfinanciación redujo esta tasa a menos de la mitad (10,5\%) (7). Pero, simultáneamente, se produjo un aumento de 12,9 puntos en la tasa de prescripciones de supresores de la tos y de 4,4 puntos para otros broncodilatadores todavía financiados. Al cabo de 4 años, la sustitución por antitusivos compensó el 55\% del ahorro del sistema de salud en mucolíticos y expectorantes. Cabe señalar que el aumento de las prescripciones de supresores de la tos es algo sorprendente desde el punto de vista clínico ya que en el caso de las tos blanda, húmeda o productiva, debe facilitarse la expectoración y solo cuando es muy intensa e impide el descanso se deben usar antitusígenos (2). A pesar de las diferencias con el caso francés, se trata de una alerta para que las autoridades vigilen las consecuencias de la desfinanciación y que no se produzca una desviación de la prescripción hacia otro tipo de medicamentos más caros, como sucedió en el pasado, o que se asista a un trasvase de un grupo no financiado a otro. Del mismo modo, existe el temor de que el médico bajo la presión del paciente, prescriba un medicamento financiado para una indicación no autorizada en ficha técnica, en lugar de otro que esté excluido del SNS, aunque este último presente dicha indicación y resulte más efectivo $\mathrm{y}$ seguro.

Los pacientes con porcentaje de aportación TSI003 normalmente po- seen un puesto de trabajo con un salario retributivo mensual y disponen de una renta inferior a $18.000 €$ al año. Por otro lado, los pacientes con TSI002 solamente ingresan su pensión y aunque se encuentran en un rango de renta similar al TSI003, presuponemos que tienen una disponibilidad económica menor que los pacientes activos, debido a que según datos del Ministerio de Empleo y Seguridad Social, la pensión media en España es de 849,60€ (8), esto es, no llega a $12.000 €$ anuales que se ve reducida si tenemos en cuenta al pensionista autónomo (598,31€ de media) (8), o recibe una pensión de viudedad (inferior a $600 €$ en más de la mitad de los casos) (9) o de otros tipos de pensiones. Además, necesitan más medicamentos y el gasto conjunto en la farmacia de dichos pacientes es notoriamente superior. La existencia de asociación estadística entre la dispensación o no del medicamento y los códigos de aportación citados, demuestra que en algunos casos una disponibilidad económica inferior conlleva no retirar estos medicamentos, lo que supone un grave problema de adherencia. Analizando los resultados, se observa que un $14 \%$ de los pensionistas no quiso los medicamentos mientras que solamente un $4 \%$ de los activos rechazó pagarlos íntegramente.

Como ha quedado reflejado en el párrafo anterior, la salida de ciertos medicamentos de la financiación podría provocar una disminución de la adherencia a los tratamientos. Esa falta de adherencia o de incumplimiento terapéutico puede derivar en el agravamiento de problemas de salud, provocando hospitalizaciones posteriores e incrementando el gasto sanitario que hoy en día tanto se quiere reducir. En nuestro estudio, un $86 \%$ de los pacientes con tratamiento crónico continuó con el mismo a pesar de tener que pagar la totalidad de su importe. La clave para que el porcentaje fuese tan elevado radicó en que los farmacéuticos realizamos una importante labor de asesoramiento y aconsejamos con insistencia la conveniencia de que continuaran con su medicación, además de que en algunos casos los médicos también habían expresado la importancia del tratamiento. Sin embargo, no siempre fue posible ya que en algunas ocasiones, manifestaron la preferencia de comentárselo previamente a su médico y en otros, preguntaron si existía alguna alternativa terapéutica que estuviera financiada. Muchas veces, ante la respuesta negativa, acababan comprándolo. Sin embargo, en otro estudio realizado por la Federación de Asociaciones en Defensa de la Sanidad Pública (FADSP) (3) se mostró que hasta un 58,3\% de los pacientes que consumían alguno de los más de 400 medicamentos que el Ministerio de Sanidad desfinanció en septiembre, dejó de hacerlo después de la entrada en vigor del Real decreto. Esta publicación solo contempló encuestas realizadas a pensionistas y no se contó con la participación de personal sanitario, por lo que podemos concluir que la información y consejo del farmacéutico comunitario es importante para minimizar la falta de adherencia, ya que extrapolando los resultados del estudio FADSP y nuestro estudio, hasta un $44 \%$ de los pacientes pensionistas continuaría el tratamiento gracias a la intervención farmacéutica, como se muestra en la figura 5.

El hecho de que en un principio se encontrara un resultado significativo entre la dispensación o no de medicamentos y la ubicación de la farmacia daba idea de que en las farmacias urbanas se rechazaba en mayor medida pagar estos medicamentos. Sin embargo, en este resultado el precio actuaba como factor de confusión. En nuestro estudio, los medicamentos de precio elevado estaban asociados en su mayoría a las farmacias urbanas. Por lo tanto, en ningún caso se halló asociación al analizar la ubicación rural o urbana, por separado, con los fármacos de precio medio y bajo, por lo que se deduce que no hay diferencias significativas en la decisión de los pacientes que viven en el pueblo o en la ciudad.

Además de asesorar, el farmacéutico tuvo que realizar en muchas ocasiones una considerable labor informadora que ralentizaba su trabajo y que conllevaba quejas por parte de los pacientes. A pesar de que en los medios de comunicación se había comunicado la noticia y de que algunos médicos se la explicaban a los pacientes cuando les prescribían uno de estos medicamentos, menos de la mitad de los pacientes eran conocedores de la implantación de este RD y sus repercusiones, demostrando 
que la notificación de la medida fue insignificante.

Para observar la evolución de los resultados de esta norma y darle más validez y representatividad al estudio, se ha planificado volver a realizarlo el año próximo en el mismo período, aunque somos conscientes de que el entorno habrá cambiado y de que posiblemente el aumento en el precio de estos medicamentos incrementará el porcentaje de pacientes que no querrán retirarlos.

\section{Conclusiones}

Los pacientes pensionistas que, presumiblemente, disponen de rentas más bajas, están más limitados a la hora de retirar los medicamentos desfinanciados ya que rechazan en mayor proporción pagar su precio íntegro. El precio del medicamento también influye a la hora de retirar o no la medicación.

Sin embargo, el número de pacientes, sobre todo activos, dispuestos a asumir el pago de estos medicamentos fue mayor que los que no aceptaron la dispensación.

El grado de adherencia a los tratamientos de continuación fue elevado, lo que denota que tanto los pacientes como los médicos consideran estos fármacos importantes.

El papel del farmacéutico comunitario tras la puesta en marcha del Real decreto ha demostrado su im- portancia en la continuidad de los tratamientos, ya que a la labor de dispensación, aporta acciones de informador y conciliador, soportando, sin ser el responsable, las quejas y críticas por parte de la población ante las nuevas medidas.

\section{Agradecimiento}

Los autores agradecen públicamente a Eduardo Satué, farmacéutico comunitario de Maella (Zaragoza) y presidente de SEFAC Aragón-Lifara, y a la doctora $\mathrm{M}^{\mathrm{a}}$ Ángeles Zabalza, jefa del servicio de farmacia del Hospital San Juan de Dios de Pamplona por su motivación, ayuda y consejos tanto a la hora de realizar el estudio como de su redacción para el artículo.

\section{Referencias bibliográficas}

1. Disposiciones generales Ministerio de Sanidad, Servicios Sociales e Igualdad. Ley 29/2006 de 26 de julio. Boletín Oficial del Estado, no 197, (17-082012).

2. Puig-Junoy J. Desfinanciar medicamentos no siempre reduce el gasto farmacéutico. Gestión Clínica y Sanitaria. 2011;13(4):152.

3. Unión Democrática de Pensionistas y Jubilados de España. Doce Causas para 2012. [monografía en Internet]. Madrid [acceso 20 febrero 2013]. Disponible en: http://www.mayoresudp. org/bddocumentos/Por-la-sostenibilidad-del-sistema-de-pensiones.pdf
4. Consumo farmacéutico por grupos terapéuticos, edad y sexo. Madrid: Instituto de Información Sanitaria; 2005.

5. Sans S, Paluzie G, Puiga T, Balañá L, Balaguer-Vintró I. Prevalencia del consumo de medicamentos en la población adulta de Cataluña. Gac Sanit. 2002;16(2):121-30. doi:10.1016/ S0213-9111(02)71643-9

6. Pichetti S, Sermet C. Le déremboursement des médicaments en France entre 2002 et 2011: éléments d'évaluation. Questions d'Économie de la Santé. 2011;167.

7. Pichetti S, Sorasith C, Sermet C. Analysis of the impact of removing mucolytics and expectorants from the list of reimbursable drugs on prescription rates: A time-series analysis for France 1998-2010. Health Policy. 2011; 102(2-3):159-69. doi:10.1016/j.healthpol.2011.07.001

8. Ministerio de Empleo y Seguridad Social. Estadísticas, presupuestos y estudios por comunidades autónomas y provincias. [monografía en Internet]. Madrid [acceso 20 febrero 2013]. Disponible en: http://www.seg-social. es/Internet_1/Estadistica/Est/Pensiones_y_pensionistas/Pensiones_contributivas_en_vigor/Por_Comunidades_Aut_nomas_y_provincias/ index.htm

9. Europa Press Salud. El $16 \%$ de los pensionistas renuncia a algún medicamento por el copago [Internet]. Lunes 25 de febrero de 2013. [acceso 26 febrero 2013]. Disponible en: http://www.europapress.es/salud/noticia-168-pensionistas-renuncia-alguno-medicamentos-nuevo-copago-20130225140712.html 